Para citar este artículo: Lopera Pareja, E. H. (2017). ¿Esto es por el cambio climático? Los fenómenos meteorológicos extremos en la prensa española (2000-2010): ocurrencia y atención mediática. Anuario Electrónico de Estudios en Comunicación Social "Disertaciones", 10(2), 79-103. Doi: http://dx.doi.org/10.12804/ revistas.urosario.edu.co/disertaciones/a.4630

\title{
¿ESTO ES POR EL CAMBIO CLIMÁTICO? LOS FENÓMENOS METEOROLÓGICOS EXTREMOS EN LA PRENSA ESPAÑOLA (2000-2010): OCURRENCIA Y ATENCIÓN MEDIÁTICA ${ }^{1}$
}

\section{Is this Climate Change? Extreme Weather Events in the Spanish Press (2000- 2010): Occurrence and Media Attention \\ É isto pela Mudança Climática? Os Fenômenos Meteorológicos Extremos na Imprensa Espanhola (2000-2010): Ocorrência e Atenção Mediática}

Emilia Hermelinda Lopera-Pareja, Centro de Investigaciones Energéticas, Tecnológicas y Medioambientales (CIEMAT)

emilia.lopera@ciemat.es

Recibido: 22 de febrero de 2016

Aceptado: 11 de julio de 2016

1 Este trabajo ha sido desarrollado con el apoyo del proyecto de investigación "Concepto y dimensiones de la cultura del riesgo", Ref. MINECO-15-FFI2014-58269-P y por la Consejería de Economía y Empleo del Principado de Asturias para "Grupo de Estudios CTS", Ref. FC-15-GRUPIN14-128. 


\section{RESUMEN}

La ocurrencia de fenómenos meteorológicos extremos (FME) ocupa el segundo puesto en la lista de riesgos derivados del cambio climático (CC), según el IPCC. Desde la aproximación de la teoría de la amplificación social del riesgo, la cobertura informativa de esta conexión causal contribuiría a "amplificar" la sensación de vulnerabilidad y exposición a estas catástrofes naturales y, en última instancia, a facilitar y agilizar las medidas de mitigación y adaptación. El objetivo de este trabajo es explorar el papel de la información periodística sobre la ciencia del clima como vector para popularizar la relación entre CC y FME. Para ello se monitorizan la ocurrencia e impactos de los FME y una muestra de informaciones sobre la ciencia del clima en la prensa española en el periodo 2000-2010. Los resultados revelan que la conexión CC-FME está presente en más de un tercio de la cobertura informativa de la ciencia del clima, bien sea asociada al aumento de las temperaturas o al impacto socioeconómico; su inclusión en el discurso mediático no depende tanto del periódico como del marco temporal, detectándose cierta permeabilidad los años en que ocurren grandes catástrofes.

Palabras clave: fenómenos meteorológicos extremos, cambio climático, cobertura informativa, vulnerabilidad, mitigación y adaptación.

\section{ABSTRACT}

The occurrence of extreme weather events (EWE) ranks second in the list of risks associated with climate change (CC) according to the IPCC. Based on the theoretical approach of the social amplification of risk, the press coverage on this causation connection would contribute to 'amplify' the feeling of vulnerability and exposure to these natural disasters and, ultimately, to facilitate and speed up mitigation and adaptation measures. The aim of this paper is to explore the role of the press coverage on climate science as a vehicle for popularizing the causation connection between CC and EWE. For this purpose, the occurrence and the impacts of the EWE along with a sample of news coverage on climate science in the Spanish press (2000-2010) are monitored. Results show that CC-EWE connection is discursively included in more than one third of the cases, either associated with the increase of temperature or with socioeconomic impacts, among others; its inclusion in the media discourse on climate science seems to depend not on the newspaper as on the time frame, since certain media permeability has been detected those years when major disasters happened.

Key words: Extreme weather events, climate change, press coverage, vulnerability, mitigation and adaptation.

\section{RESUMO}

A ocorrência de fenômenos meteorológicos extremos (FME) ocupa o segundo lugar na lista de riscos derivados da mudança climática (CC), segundo o IPCC. Desde a aproximação da teoria da amplificação social do risco, a cobertura informativa desta conexão causal contribuiria a 'amplificar' a sensação de vulnerabilidade e exposição a estas 


\section{DISERTACIONES}

ESTUDIOS

Anuario electrónico de estudios en Comunicación Social

ISSN: $1856-9536$

Doi: http://dx.doi.org/10.12804/revistas.urosario.edu.co/disertaciones/a.4630

Volumen 10, Número 2 / Julio-diciembre 2017

Versión PDF para imprimir desde

http://revistas.urosario.edu.co/index.php/disertaciones

catástrofes naturais e, em última instância, a facilitar e agilizar as medidas de mitigação e adaptação. 0 objetivo deste trabalho é explorar o rol da informação jornalística sobre a ciência do clima como vetor para popularizar a relação entre CC e FME. Para isso se monitoriza a ocorrência e impactos dos FME e uma mostra de informações sobre a ciência do clima na imprensa espanhola no período 2000-2010. Os resultados revelam que a conexão CCFME está presente em mais de um terço da cobertura informativa da ciência do clima, bem associada ao aumento das temperaturas ou ao impacto socioeconómico; a sua inclusão no discurso mediático não depende tanto do jornal quanto do marco temporal, se detectando certa permeabilidade os anos em que ocorrem grandes catástrofes.

Palavras-chave: fenômenos meteorológicos extremos, mudança climática, cobertura informativa, vulnerabilidade, mitigação e adaptação.

\section{Introducción}

El aumento de frecuencia y/o intensidad de los fenómenos meteorológicos extremos (FME) relacionados con el cambio climático (CC) es una cuestión que suscita preocupación en el ámbito científico (IPCC, 2014; ONISDR, 2015), económico (World Economic Forum, 2016) y social (Carle, 2015), aunque aún existen interrogantes sobre la naturaleza de esta relación causa-efecto en los que se sigue investigando (WWA, 2016). Con la expresión "fenómenos meteorológicos extremos" se hace referencia a los eventos adversos de las tres primeras categorías de desastres naturales establecidas por el Centro de Investigación sobre la Epidemiología de los Desastres (CRED) y recogidas en el informe The Human Cost of Weather Related Disasters 1995-2015 (CRED, 2016): fenómenos hidrológicos, meteorológicos y climatológicos, entre los que se incluyen inundaciones, sequías y fuertes tormentas, entre otros (tabla 1).

La década 2001-2010 ha sido la más cálida registrada desde 1850 y dicho aumento de temperatura ha coincidido con una ocurrencia de FME sin precedentes, según la Organización Meteorológica Mundial (ONISDR, 2015). El aumento de la frecuencia de ocurrencia y/o intensidad de dichos fenómenos ocupa el segundo puesto como motivo de preocupación en la lista de los cinco riesgos relacionados con el CC que, como tales, aparecen recogidos en el V Informe de Evaluación del Panel de Expertos de la ONU sobre Cambio Climático (IPCC, 2014). Pero las llamadas de atención no solo provienen de la esfera científica. En el Informe sobre Riesgos Globales 2016, realizado por los organizadores del Foro Económico Mundial de Davos, dos riesgos asociados al CC se sitúan por primera vez en los primeros puestos: 1) el fracaso de las medidas de mitigación y adaptación al CC, en términos de impacto; y 2) la 
ocurrencia de FME, en términos de probabilidad (World Economic Forum, 2016, p. 11)². En cuanto a la percepción social del fenómeno, las encuestas internacionales realizadas por el Pew Research Center revelan que el CC también es percibido como una de las mayores amenazas globales, la más preocupante para los ciudadanos de Latinoamérica y África y la segunda más preocupante, por detrás de ISIS, en Europa y Oriente Próximo (Carle, 2015).

Tabla 1. Clasificación de los desastres naturales según el Centro de Investigación sobre la Epidemiología de los Desastres (CRED, 2016)

\begin{tabular}{|c|c|c|c|c|c|}
\hline \multicolumn{6}{|c|}{ Tipos de desastres naturales } \\
\hline Hidrológicos & Meteorológicos & Climatológicos & Geofísicos & Biológicos & Extraterrestres \\
\hline $\begin{array}{c}\text { Inundaciones } \\
\text { Corrimientos de } \\
\text { tierra } \\
\text { Temporal } \\
\text { marítimo y fuerte } \\
\text { oleaje }\end{array}$ & $\begin{array}{l}\text { Temporales } \\
\text { Temperaturas } \\
\text { extremas } \\
\text { Niebla }\end{array}$ & $\begin{array}{c}\text { Sequías } \\
\text { Inundaciones por } \\
\text { deshielo de lagos } \\
\text { glaciares } \\
\text { Incendios }\end{array}$ & $\begin{array}{c}\text { Terremotos } \\
\text { Corrimientos de } \\
\text { tierra (sin lluvias) } \\
\text { Actividad } \\
\text { volcánica }\end{array}$ & $\begin{array}{l}\text { Con animales } \\
\text { Epidémicos } \\
\text { Plagas de insectos }\end{array}$ & $\begin{array}{l}\text { Impactos } \\
\text { Meteorología } \\
\text { espacial }\end{array}$ \\
\hline
\end{tabular}

Fuente: CRED (2016). Elaboración propia.

Dentro de los estudios de la comunicación del riesgo, la teoría de la amplificación social de riesgo (Renn, 1991; Renn et al., 1992) mantiene que la forma en que las personas perciben y reaccionan ante los riesgos, es decir, su "experiencia social del riesgo", no depende exclusivamente de la denominada evaluación técnica del riesgo -concebida como el producto de la probabilidad de ocurrencia y la magnitud de las consecuencias-, sino que también depende de otras cinco variables que amplifican o atenúan dichos riesgos: las consecuencias físicas, la percepción individual del ciudadano no experto, su reacción ante la amenaza, el impacto socioeconómico y político y, por último, la cantidad de cobertura informativa. Desde la aproximación de este marco conceptual, que considera el volumen de atención mediática como uno de sus factores clave, el presente trabajo explora el papel vehicular de la cobertura informativa de la ciencia del clima en la construcción social del riesgo climático mediante la difusión de la existencia de un nexo entre el CC y la ocurrencia de FME. Aplicando el modelo teórico de estos autores (Renn et al., 1992) al estudio del caso, se podría decir que la popularización de la conexión CC-FME, a través de los medios de comunicación, actuaría sobre la experiencia social del riesgo aumentando la sensación de vulnerabilidad ante estos efectos adversos. De acuerdo con este paradigma, esta podría ser una forma de agilizar la acción política frente al CC y mejoraría su legitimación (González Alcaraz, 2014).

Desde el punto de vista metodológico, la exploración de esta cuestión se podría abordar mediante dos fórmulas: considerando la cobertura informativa de los FME como vector de las alusiones al CC, como ya se ha hecho (Gavin et al., 2011; Pasquare y Oppizzi, 2012), o bien considerando la cobertura informativa del CC como vehículo en el que se incorporan contenidos sobre FME fuera de las "quiebras del acontecer" provocadas por desastres concretos. Tomando como base teórica los trabajos de Lozano Ascencio, Piñuel Raigada y Gaitán Moya sobre el

2 Se trata de un informe anual que, en su undécima edición, presenta los resultados de una encuesta realizada a 750 expertos y políticos de todo el mundo sobre percepción de 29 riesgos globales -sociales, tecnológicos, económicos, medioambientales y políticos- para la próxima década. 
discurso mediático (como, por ejemplo, Lozano Ascencio et al., 2012), en esta investigación se adopta el segundo planteamiento. Con ello se intenta soslayar el discurso mediático de los FME como meras narraciones de tales "quiebras del acontecer", caracterizadas por un alto nivel de urgencia, atención mediática limitada en el tiempo y escasa reflexión sobre el origen del riesgo y sobre los factores de vulnerabilidad. Teniendo en cuenta las anteriores consideraciones, el objetivo de este trabajo es la monitorización de la ocurrencia de FME en el mundo y en España (Objetivo 1), y la monitorización del papel de la cobertura informativa de la ciencia del clima en la prensa española como vector informativo de los FME asociados al CC (Objetivo 2).

Mientras que con el Objetivo 1 se busca dotar a la investigación de un contexto global y nacional caracterizado por un aumento continuado de los FME y de sus consecuencias, con el Objetivo 2 se persigue determinar el papel de los medios de comunicación a la hora de amplificar dichos riesgos y relacionarlos causalmente con el CC.

\section{Antecedentes y estado de la cuestión}

\section{Los fenómenos meteorológicos extremos y el cambio climático}

Para los expertos en física del aire, los fenómenos extremos están integrados en el clima, si bien es cierto que con una baja probabilidad de ocurrencia (Almarza Mata, 2001). Los estudios que analizan la relación entre el CC antropogénico y la ocurrencia de FME, como tormentas, inundaciones, olas de calor y sequías, sigue movilizando a la comunidad científica. Analizar esta relación y comunicarla a los gestores políticos y al público es el objetivo del proyecto World Weather Attribution, de la organización sin ánimo de lucro Climate Central ${ }^{3}$. Según se recoge en su página web (WWA, 2016), "la identificación de la huella humana en cada uno de los eventos meteorológicos extremos, mediante atribución probabilística, ha sido un objetivo importante para la comunidad científica desde hace más de una década".

El aumento de la evidencia científica que apunta a la existencia de una relación entre el CC antropogénico y el aumento de la frecuencia e intensidad de FME ha servido de estímulo para la realización de estudios sobre el impacto económico y social que dichos eventos podrían originar. Así se ha investigado la reubicación de empresas como una estrategia de adaptación al CC y a los FME (Linnenluecke y Griffiths, 2010; Linnenluecke et al., 2011). Según estos trabajos, las consecuencias del CC en la meteorología pueden ser de dos tipos: ocurrencia de FME o cambios graduales manifestados, por ejemplo, en un aumento de la temperatura media. Aunque los primeros suelen afectar con mayor severidad a los sistemas humanos en un plazo muy corto de tiempo, los segundos pasan desapercibidos, aunque también tienen un gran impacto a más largo plazo en el suministro de alimentos, en los recursos hídricos, en los ecosistemas e incluso en los sistemas de circulación atmosférica y oceánica (Scheffer et al., 2001).

Otra cuestión tratada es la necesidad de reducir el riesgo de desastres naturales integrando la información disponible sobre los FME derivados del CC junto con otros factores de riesgo, como la vulnerabilidad de los sistemas

3 El proyecto WWA se puso en marcha a finales de 2014 como fruto del acuerdo entre Climate Central, la Universidad de Oxford, la Universidad de Melbourne, el Royal Netherlands Meteorological Institute y el Centro del Clima de la Cruz Roja Internacional. 
afectados por el CC (Van Aalst, 2006). Según él, este análisis es especialmente importante en vista de la creciente vulnerabilidad ante los desastres naturales de acuerdo con el aumento del número de personas afectadas y de los costes económicos de los daños causados.

Aunque los estudios sobre la percepción social de la información meteorológica han sido escasos en España (Crespo et al., 2014; Sánchez Calero, 2005), el último de estos trabajos ha puesto de manifiesto que existe una divergencia entre las condiciones meteorológicas reales y las percibidas, lo que "abre una importante pregunta a indagar de modo empírico sobre la percepción y la comunicación en meteorología y cambio climático" (Crespo et al., 2014, p. 190). Pero las confusiones no son exclusivas del público lego. Mediante una encuesta a más de doscientos "hombres y mujeres del tiempo" de las televisiones locales estadounidenses, Wilson (2002) analizó el papel de los programas de información meteorológica como fuente de información del CC. Así comprobó que entre este colectivo existe un amplio desconocimiento y una desinformación sobre los fundamentos elementales de la ciencia del clima, lo que estaba relacionado, en parte, con los valores y creencias de los presentadores sobre el CC.

La percepción social de los FME como manifestaciones del CC es una cuestión por la que ya se ha preguntado en varias ocasiones a los ciudadanos españoles (Meira et al., 2013; Meira et al., 2011; Meira et al., 2009). En las tres encuestas de los citados autores se plantearon dos ítems sobre este asunto: el primero de ellos, sobre percepción de las condiciones meteorológicas en la actualidad (Q20) y, el segundo, en el horizonte temporal de los próximos veinte años (Q21). Con independencia de su lugar de residencia, al ser preguntados por la meteorología actual, más del $60 \%$ de los encuestados está muy de acuerdo o bastante de acuerdo con la afirmación "Las tormentas y los temporales son ahora más frecuentes que antes", aunque el mayor nivel de acuerdo (83 \%) se alcanzó con el aserto "Antes había estaciones y ahora se notan menos". En el horizonte temporal para las próximas dos décadas, los encuestados creen (muy probable o bastante probable) la sucesión de periodos de sequía más frecuentes (86\%), el aumento de los incendios forestales ( $85 \%)$, el aumento significativo de las temperaturas (84\%) y el incremento de las inundaciones (75 \%) (Meira et al., 2013, p. 60 y ss.).

\section{Fenómenos meteorológicos extremos, cambio climático y cobertura informativa}

Aunque con cierto retardo en comparación con el ámbito anglosajón (Boykoff y Roberts, 2007), en la actualidad se puede afirmar que la investigación sobre la cobertura informativa del CC ha adquirido cierta madurez en España, principalmente durante el último lustro, gracias a la financiación de varios proyectos de investigación en el ámbito universitario y de los organismos públicos de investigación. El resultado de estos proyectos se ha materializado en la publicación de varios libros (Fernández Reyes y Mancinas Chávez, 2013; León, 2014; Piñuel Raigada et al., 2012), tesis doctorales (De Rueda Úbeda, 2015; Lopera, 2013; Teso-Alonso, 2016), artículos y presentaciones en congresos. Durante este recorrido se ha explorado la información periodística del CC en España mediante estudios longitudinales que monitorizan un periodo más o menos largo, bien en momentos clave asociados a determinados acontecimientos, como la presentación de los informes de evaluación del IPCC o la celebración de las cumbres mundiales sobre el clima, o bien en momentos no asociados a ningún acontecimiento. En este último caso se trataba de analizar el tratamiento informativo del CC "en tiempos de calma" (Lozano Ascencio, 2013).

El hecho de que los medios de comunicación se hagan eco del posible nexo entre el CC y los FME puede ser un argumento para legitimar y acelerar la toma de decisiones para hacer frente al fenómeno mediante medidas 
de mitigación y adaptación. Por tanto, que la prensa conecte CC y FME podría interpretarse como una manera de realizar una llamada de atención a la ciudadanía sobre la necesidad de actuar frente al fenómeno y hacerlo rápido. Existe una serie de trabajos que, desde distintas aproximaciones teóricas y metodológicas, y aunque sea tangencialmente, abordan esta cuestión.

En este sentido ya ha sido puesto de manifiesto que los medios de comunicación son la fuente de información preferida y la que más puede influir en la comprensión de fenómenos catastróficos pues, incluso en zonas fuertemente castigadas por sucesos meteorológicos extremos solo el 50 \% de los afectados identificó la observación personal directa como fuente de información (Biernacki et al., 2008). Esto está en línea con Lozano Ascencio (2013, p. 134), que explica que "los relatos mediáticos de catástrofes [...] se llegan a convertir en unas poderosas 'claves narrativas' para que las audiencias sean capaces de construir (entender, relacionar, memorizar) la realidad alterada".

Desde esta perspectiva, Gavin et al. (2011) analizaron con qué frecuencia y cómo aparece la conexión causa-efecto entre el CC y las inundaciones en una selección de periódicos británicos en el periodo 2001-2007. Concluyeron que la información sobre inundaciones no era la plataforma más adecuada para aumentar la relevancia del CC por varias razones. En primer lugar, por su reducida atención mediática en comparación con otros temas cotidianos como la política, la economía y la salud; en segundo lugar, porque dichas noticias tienden a destacar más los aspectos relacionados con el interés humano o el impacto económico y la cobertura de las aseguradoras, que la relación entre las inundaciones y el CC; y, por último, en las noticias en que se establece dicha conexión, se lanzan mensajes contradictorios que, en algunas ocasiones, crean incertidumbre científica. En otro estudio se ha comprobado que el grado de atención mediática a los desastres naturales depende de las características y dimensiones de dicho evento, es decir, de su mayor o menor severidad, y no tanto de la distancia geográfica o del país en el que ocurra (Yan y Bissell, 2015).

Al otro lado del Atlántico, a partir de la ocurrencia del huracán Sandy, en Estados Unidos, en 2012, y de las inundaciones sufridas el año siguiente, en Canadá, la prensa de estos países comenzó a contextualizar las medidas de adaptación al CC como una manera de prepararse ante el acaecimiento de FME (Ford y King, 2015). Por el contrario, en el caso de la prensa italiana y su cobertura informativa de los fenómenos hidrológicos, la atención se centró más en las consecuencias del desastre que en las medidas de prevención (Pasquare y Oppizzi, 2012). Estos autores también comprobaron que la tendencia de la prensa italiana a confundir meteorología con clima, culpando al CC de las lluvias torrenciales que causaron graves inundaciones y deslizamientos de tierra, disminuyó de 2007 a 2010. Pero no en todos los países las condiciones meteorológicas extremas son un buen indicador de la atención dedicada al CC (Schaefer et al., 2014; Ungar, 1999), pues eventos como las celebraciones de las cumbres del clima anuales o iniciativas de organizaciones no gubernamentales para construir la agenda han tenido un mayor impacto (Schaefer et al., 2014).

Mientras que la cobertura informativa del CC ha descendido desde finales de la pasada década, tanto en España (Fernández Reyes et al., 2015) como en el ámbito anglosajón (Brulle et al., 2012), por el contrario, la atención mediática a los FME ha aumentado (Nerlich y Jaspal, 2014).

En lo que se refiere a la investigación sistemática de FME en la prensa española, pero fuera del marco de la cobertura informativa sobre el CC, cabe citar el estudio llevado a cabo por León Gross y Ruiz Sinoga (2012) en el que ponen de manifiesto que existe un alto grado de coherencia entre los episodios de sequía extrema en el sur de 
España y el volumen de atención mediática dedicado a dicho fenómeno, especialmente con las sequías severas sufridas en la zona en los años ochenta y noventa.

En el contexto español, la meteorología actual como elemento narrativo en la cobertura informativa de la ciencia del clima se ha revelado como uno más de una serie de aspectos secundarios en este tipo de informaciones $^{4}$, junto con los aspectos económicos y las políticas nacionales, entre otros (Lopera, 2013). También se ha explorado la imbricación de los FME en el discurso mediático del CC en España a través de los elementos visuales que ilustran las informaciones (Lopera y Moreno, 2014a), aspecto también investigado en otros países del ámbito anglosajón (Nerlich y Jaspal, 2014). Sin embargo, más allá del análisis de la meteorología actual como elemento narrativo y del análisis iconográfico, en España parece existir cierta laguna sobre el papel de la cobertura informativa sobre CC como vector para popularizar la conexión CC-FME.

El presente trabajo pretende ser una primera aproximación para salvar, al menos en parte, dicha laguna y contribuir al conocimiento disponible sobre este tema en el marco de los estudios sobre comunicación del riesgo, una cuestión relevante para la gestión del riesgo y de plena vigencia académica5.

\section{Método}

El diseño de este trabajo persigue dos objetivos:

- Objetivo 1. Monitorización de la ocurrencia de FME y sus consecuencias a nivel global y nacional.

- Objetivo 2. Monitorización de la cobertura informativa de la ciencia del clima como vector informativo de los FME asociados al CC.

Para la monitorización de la ocurrencia de FME y sus consecuencias (Objetivo 1) se ha utilizado la base de datos mundial sobre desastres del Centro de Investigación sobre la Epidemiología de los Desastres (CRED) . Para que un desastre quede registrado en dicha base de datos debe cumplir, al menos, uno de los siguientes criterios: 1) el fallecimiento de diez o más personas; 2) que haya afectado a cien o más personas; 3) que se haya declarado el estado de emergencia; 4) que se haya solicitado ayuda internacional (CRED, 2016). Con los datos procedentes de esta fuente, se acometen los siguientes análisis:

1. Evolución de la ocurrencia de FME en el mundo en el periodo 1900-2014.

2. Evolución de la ocurrencia de FME en España en el periodo 2000-2010 por categorías y su correspondiente coste económico.

Para el análisis del papel de los contenidos informativos sobre el CC como vector de difusión del posible nexo entre dicho fenómeno y los FME (Objetivo 2), se recurre a la base de datos en Access de una investigación longitu-

4 Este resultado se obtuvo mediante el análisis de escalamiento multidimensional con el paquete estadístico SPSS.

5 Este es el caso del proyecto Effective Two-Way Communications of Geohazards de la Universidad de Manchester que tiene como objetivo mejorar la eficacia de la comunicación bidireccional entre las comunidades expuestas a FME relacionados con el cambio climático y los expertos en riesgos geológicos, de manera que ambas comunidades salgan beneficiadas.

6 Disponible en http://www.emdat.be, se coordina y actualiza desde la Universidad Católica de Lovaina, aunque en sus inicios contó con el apoyo de la Organización Mundial de la Salud y del Gobierno belga. 
dinal sobre la cobertura informativa de la ciencia del clima en la prensa española en los diarios El País, El Mundo, $A B C$, Expansión y Levante en el periodo 2000-2010. Dicha base de datos recoge los resultados codificados de un análisis de contenido cuantitativo (Krippendorff, 1990; Riffe et al., 2005) aplicado a una muestra de 363 ítems y realizado por la misma autora del presente estudio para su tesis doctoral (Lopera, 2013) 7 .

El presente trabajo pretende explotar los datos del citado análisis de contenido correspondientes a una submuestra de 124 unidades de análisis de la muestra inicial, cuyo criterio de selección fue que todos los ítems mencionaran la ocurrencia de FME como consecuencia del CC, bien de manera genérica o refiriéndose a algunos de los fenómenos recogidos en la tabla 1. Sobre dicha submuestra se realizan los siguientes análisis:

1. Contextualización de los FME en la taxonomía general de las consecuencias del CC.

2. Relación de los FME con otras consecuencias del CC.

3. Evolución de los FME en la cobertura informativa del CC por años y por periódicos.

4. Recurrencia de los FME en función de otras variables: fuentes de información, escala espacial y temporal de las consecuencias y enmarcado de la ciencia del clima como ciencia sólida o incierta.

\section{Resultados}

\section{Objetivo 1. Monitorización de la ocurrencia de FME y sus consecuencias a nivel global y nacional}

\section{Evolución de la ocurrencia de FME en el mundo en el periodo 1900-2014}

La ocurrencia a nivel mundial de FME ha ido aumentando progresivamente desde la mitad del siglo XX hasta la actualidad (figura 1). Sin embargo, hay que destacar que en los últimos treinta años el aumento de la frecuencia ha sido vertiginoso en el caso de los fenómenos extremos de carácter hidrológico y meteorológico, de tal forma que, en algunos años de la última década, los valores de los años ochenta se han multiplicado por cuatro y por cinco.

La figura 2, a modo de lupa, representa una visión ampliada de la evolución de los distintos FME en lo que llevamos de siglo. Por subtipos, los FME más frecuentes en todo el mundo en los últimos quince años son las inundaciones, seguidas de los temporales y tormentas y, a mucha distancia, las sequías.

Además de las pérdidas en vidas humanas y del número de personas afectadas, los desastres naturales ocasionados por los FME tienen un alto coste económico. En el Informe de Naciones Unidas sobre Evaluación Global de la Reducción del Riesgo de Desastres (ONISDR, 2015) se menciona que los modelos de simulación utilizados indican que el riesgo del impacto económico aumenta conforme se incrementa el valor de los bienes expuestos a dichos riesgos. Esto significa que los países industrializados, con más y mejores infraestructuras, también tienen que hacer frente a un coste económico más alto si, por ejemplo, dichas infraestructuras se ven afectadas. En esa

7 Para información detallada sobre el conjunto de variables y el proceso de codificación se puede consultar el capítulo sobre el método del siguiente trabajo (Lopera, 2013, p. 93 y ss.), disponible en http://roderic. uv.es/bitstream/handle/10550/27416/TESIS\%20COMPLETA_04_11_2013.pdf?sequence=1 


\section{DISERTACIONES}

ESTUDIOS

Figura 1. Ocurrencia de fenómenos meteorológicos extremos en el mundo de 1900 a 2014

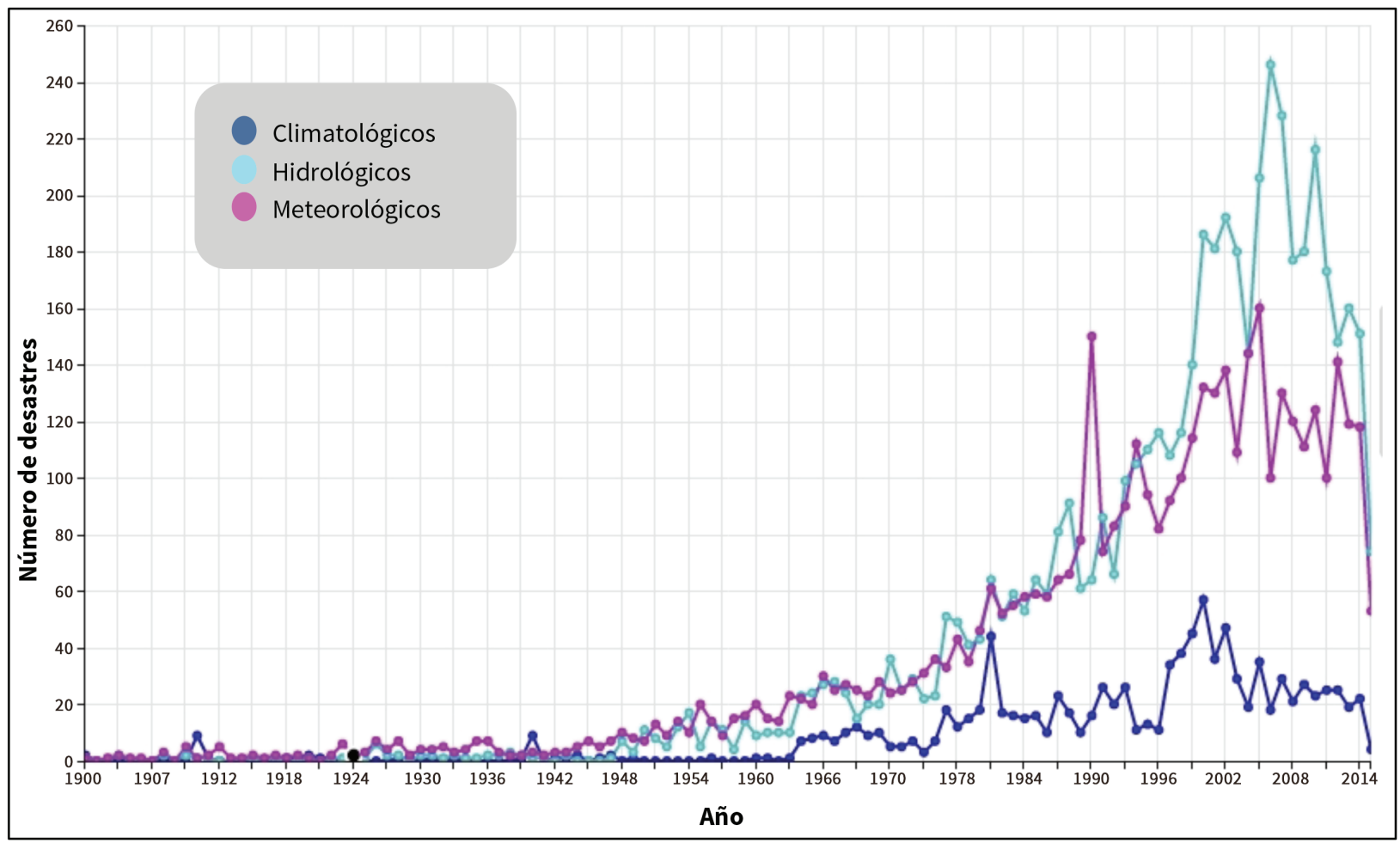

Fuente: Gráfico generado con la aplicación y los datos de la Base de Datos Internacional sobre Desastres (EM-DAT, 2016).

misma línea, otro trabajo concluye que el aumento de las pérdidas económicas originadas en Estados Unidos por los FME no se debe exclusivamente al CC sino también a los cambios sociales experimentados durante las últimas décadas (Changnon, 2003).

El histograma representado en la figura 3 muestra la evolución de los costes económicos a nivel mundial en el periodo 2000-2015 en función del tipo de FME. Como se puede apreciar, la mayor parte de los costes económicos se debe a la ocurrencia de tormentas y temporales de gran intensidad, seguidos de los daños materiales causados por las inundaciones y, por último, las sequías. En términos generales, el año 2005 fue el ejercicio con mayores pérdidas económicas del periodo analizado, superando los 240000 millones de dólares estadounidenses. Gran parte de dicho importe corresponde a los daños ocasionados por el huracán Katrina, que asoló la costa Este estadounidense a finales de agosto de 2005, cuyo coste estimado ronda los 200000 millones de dólares (Congleton, 2006). 
Figura 2. Ocurrencia de inundaciones, temporales y sequías en el mundo de 2000 a 2015

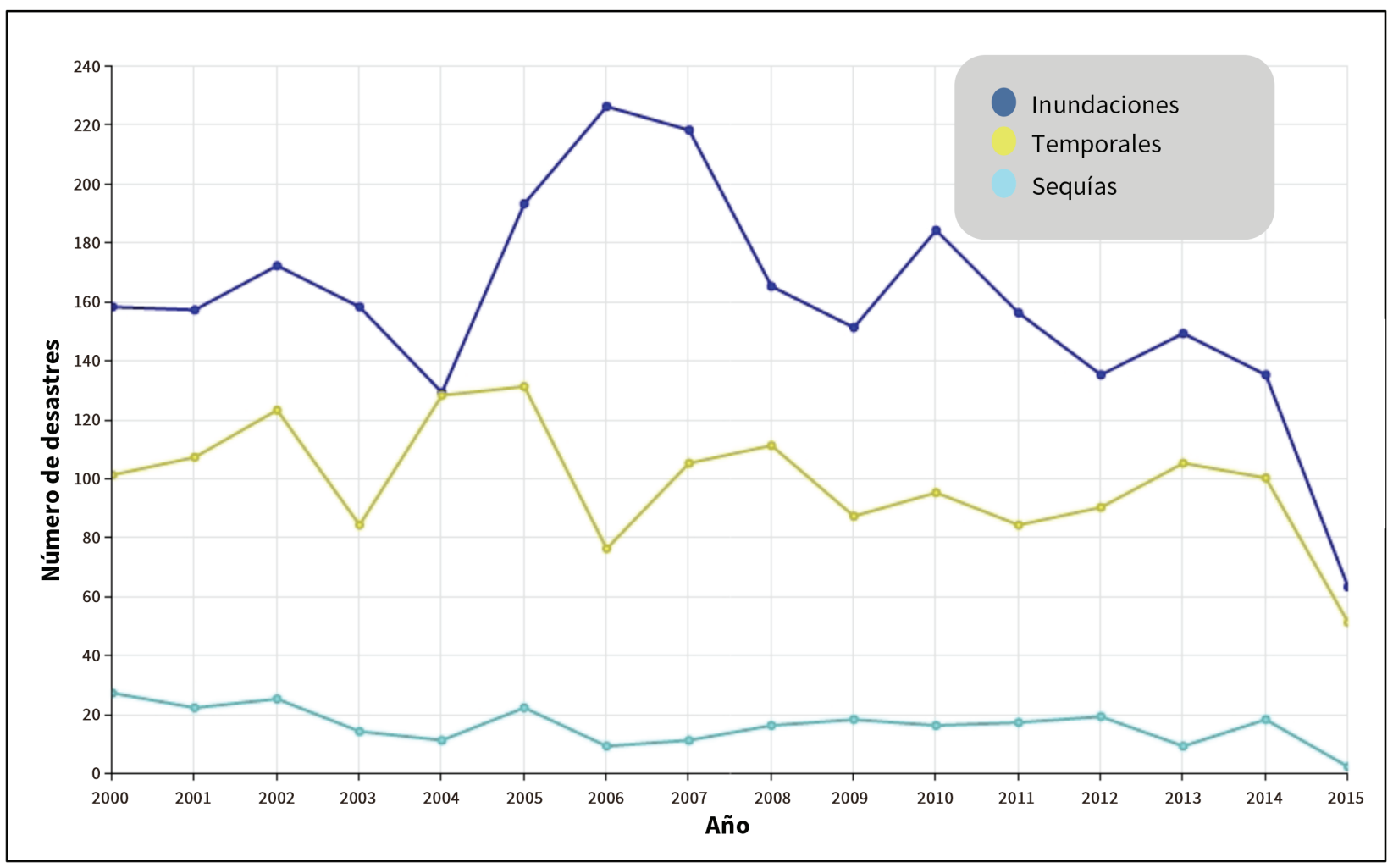

Fuente: Gráfico generado con la aplicación y los datos de la Base de Datos Internacional sobre Desastres (EM-DAT, 2016). 
Figura 3. Coste económico de los daños causados por inundaciones, temporales y sequías en todo el mundo de 2000 a 2010. Cifras en millones de dólares estadounidenses revalorizados a fecha de 2014

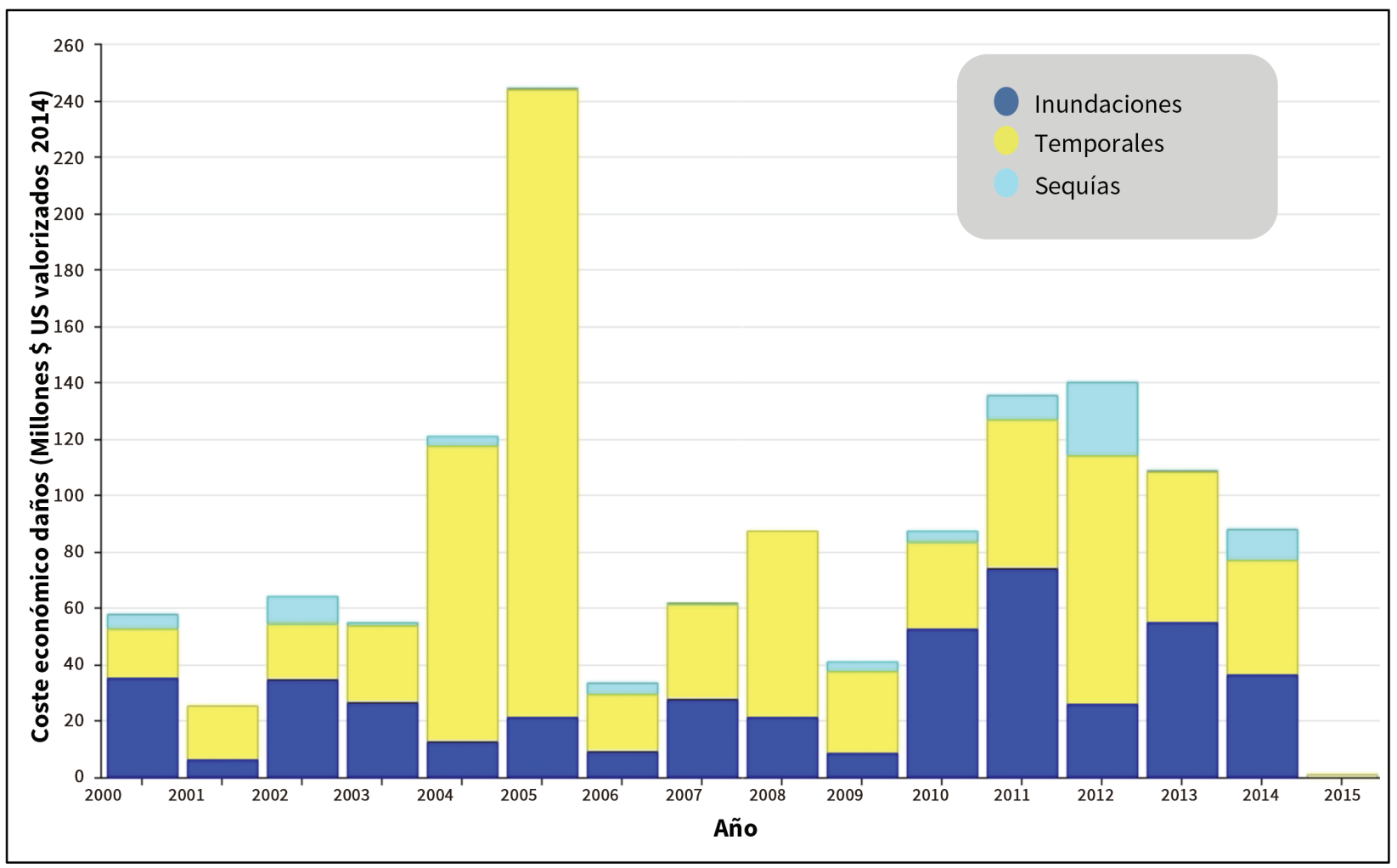

Fuente: Gráfico generado con la aplicación y los datos de la Base de Datos Internacional sobre Desastres (EM-DAT, 2016).

\section{Evolución de la ocurrencia de FME en España en el periodo 2000-2010 por categorías y coste económico}

La Base de Datos Internacional sobre Desastres Naturales (EM-DAT, 2016) registró en España la ocurrencia de 25 fenómenos extremos entre 2000 y 2010, a consecuencia de los cuales fallecieron más de 15000 personas y se vieron afectadas casi 7500 más (ver tabla 2). Aunque los episodios más frecuentes fueron las inundaciones, al igual que ocurre en el resto del mundo, la inmensa mayoría de las víctimas mortales falleció a causa de la ola de calor que azotó la Península (especialmente Andalucía) en agosto de 2003. El mayor número de personas afectadas se alcanzó debido a inundaciones e incendios forestales, mientras que los incendios forestales y las tormentas tropicales tuvieron mayor coste económico. El coste económico total en España para toda la década rondó los 6500 millones de dólares. 
En el caso de los incendios forestales, al daño en términos económicos hay que añadir el daño ocasionado al patrimonio natural, mucho más difícil de cuantificar. La ocurrencia de FME y los denominados "días de altas temperaturas", dos variables estrechamente relacionadas con el CC, se asocian al aumento de los incendios forestales (Padrón Castañeda y Barranco Retes, 2014).

Tabla 2. Desastres naturales ocurridos en España entre 2000 y 2010, cifras de fallecidos, afectados y costes económicos

\begin{tabular}{|l|l|c|c|c|c|}
\hline \multicolumn{1}{|c|}{ Tipo de desastre } & \multicolumn{1}{|c|}{ Subtipo } & Eventos & Fallecidos & Afectados & $\begin{array}{c}\text { Coste económico } \\
\text { (millones de \$) }\end{array}$ \\
\hline Incendio forestal & & 6 & 29 & 1301 & 2712 \\
\hline Inundación & Riada, crecida & 12 & 61 & 6112 & 648 \\
\hline $\begin{array}{l}\text { Temperaturas } \\
\text { extremas }\end{array}$ & Ola de calor & 3 & 15137 & 0 & 880 \\
\hline $\begin{array}{l}\text { Temperaturas } \\
\text { extremas }\end{array}$ & Frío y nieve & 1 & 9 & 0 & 0 \\
\hline Tormenta & Tormenta tropical & 2 & 17 & 0 & 240 \\
\hline Tormenta & Tormenta convectiva & 1 & 1 & 0 & 0 \\
\hline TOTAL & & 25 & 15254 & 7413 & 6480 \\
\hline
\end{tabular}

Fuente: Elaboración propia a partir de la aplicación y los datos de Base de Datos Internacional sobre Desastres (EM-DAT, 2016).

Por zonas geográficas, las temperaturas extremas de las olas de calor afectaron fundamentalmente a Andalucía, mientras que las condiciones invernales extremas se dieron en el norte, noreste y sureste de España. Las grandes tormentas o temporales se registraron en Galicia, Asturias, Cantabria, País Vasco, Cataluña, Castilla y León, Castellón, Mallorca y Menorca. En cuanto a inundaciones e incendios, España presentó un alto grado de vulnerabilidad en toda su geografía en la década pasada, pues de 2000 a 2010 se produjeron inundaciones importantes en Cataluña, Castellón, Valencia, Murcia, Zaragoza, Teruel, Albacete, Tenerife, Málaga, Galicia, Huesca, Huelva, Madrid, Islas Baleares y Sevilla. Los incendios más importantes ocurrieron en Galicia, Castilla-León, Cataluña, Cuenca, Huelva, Extremadura, Sevilla, Murcia, Teruel, Almería y Guadalajara.

\section{Objetivo 2. Monitorización de la cobertura informativa de la ciencia del clima como vector informativo de los FME asociados al CC}

\section{Los FME en la taxonomía de las consecuencias del CC}

La ocurrencia de FME es el segundo impacto más frecuente de la información periodística sobre la ciencia del clima en la prensa española (34 \%; 124 casos); a mucha distancia del aumento medio de las temperaturas (64 \%; 231 casos), que casi lo duplica, y ligeramente por encima de la subida del nivel del mar, las consecuencias medioambientales y socioeconómicas, entre otras (figura 4). 


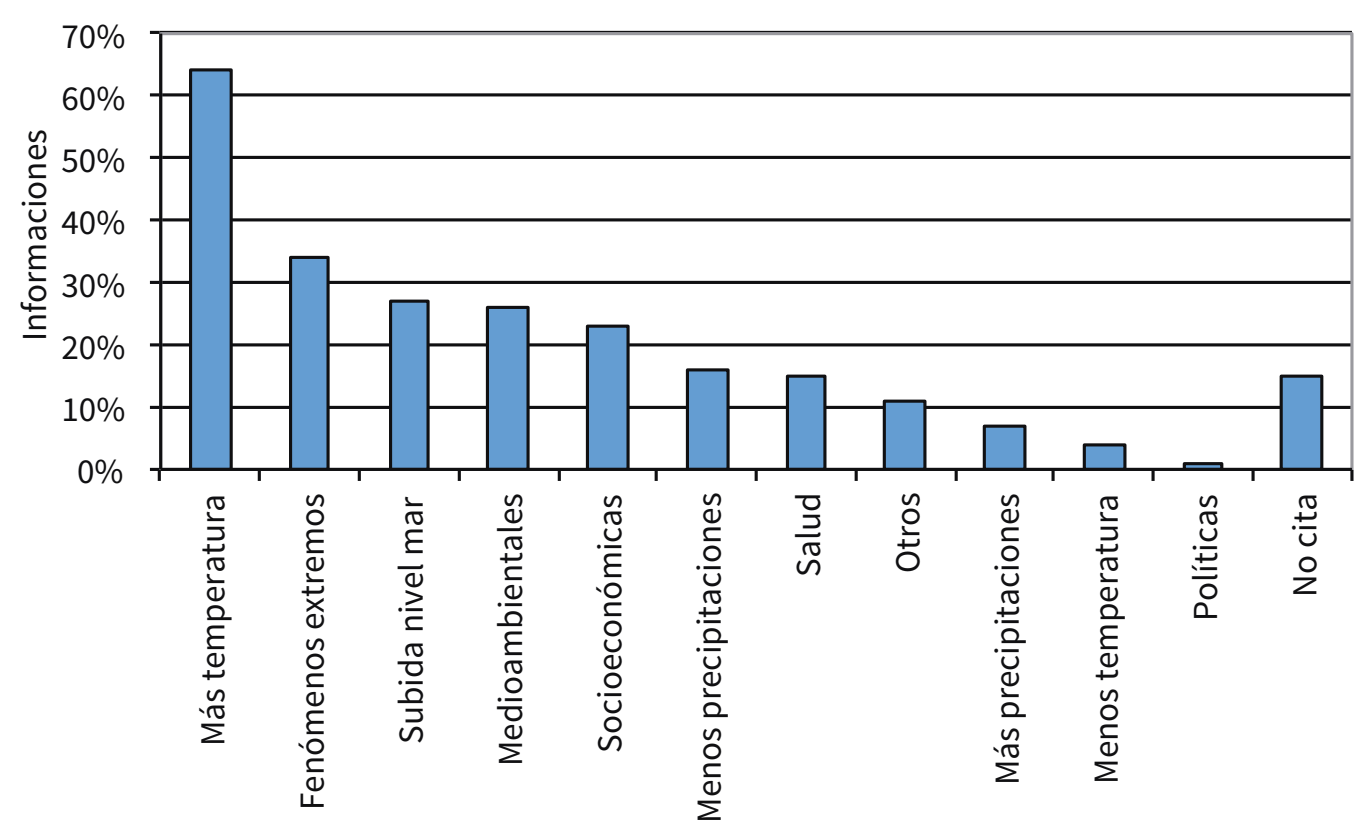

Fuente: Lopera (2013).

\section{Contextualización de los FME con otras consecuencias del CC}

Las alusiones a los FME en la cobertura informativa de la ciencia del clima prácticamente no aparecen en solitario, sino que en el $99 \%$ de los casos se mencionan junto a otras consecuencias del CC con las que se asocian. En este sentido, la combinación más frecuente que se ha hallado es "FME y aumento de las temperaturas" (75\%), lo que revela que este tándem de impactos es, con diferencia, el más recurrente a la hora de ilustrar las consecuencias del CC en la cobertura informativa de la ciencia del clima en la prensa española en el periodo analizado (figura 5).

Este mismo gráfico muestra que las consecuencias socioeconómicas son el segundo tipo de impacto que más se asocia a la ocurrencia de FME en prácticamente cuatro de cada diez informaciones. Si comparamos el resultado de la figura 4 (taxonomía general) con el de la figura 5 (categorías asociadas a FME), se advierte que mientras en la clasificación general de las consecuencias del CC el impacto socioeconómico ocupa el quinto puesto, como categoría asociada a los FME asciende en el ranking al segundo lugar. Respecto a las precipitaciones, los FME son más frecuentes en informaciones en que también se habla del descenso de las lluvias que, en periodos prolongados, da lugar a las sequías. 
Figura 5. Consecuencias del cambio climático asociadas a la ocurrencia de FME

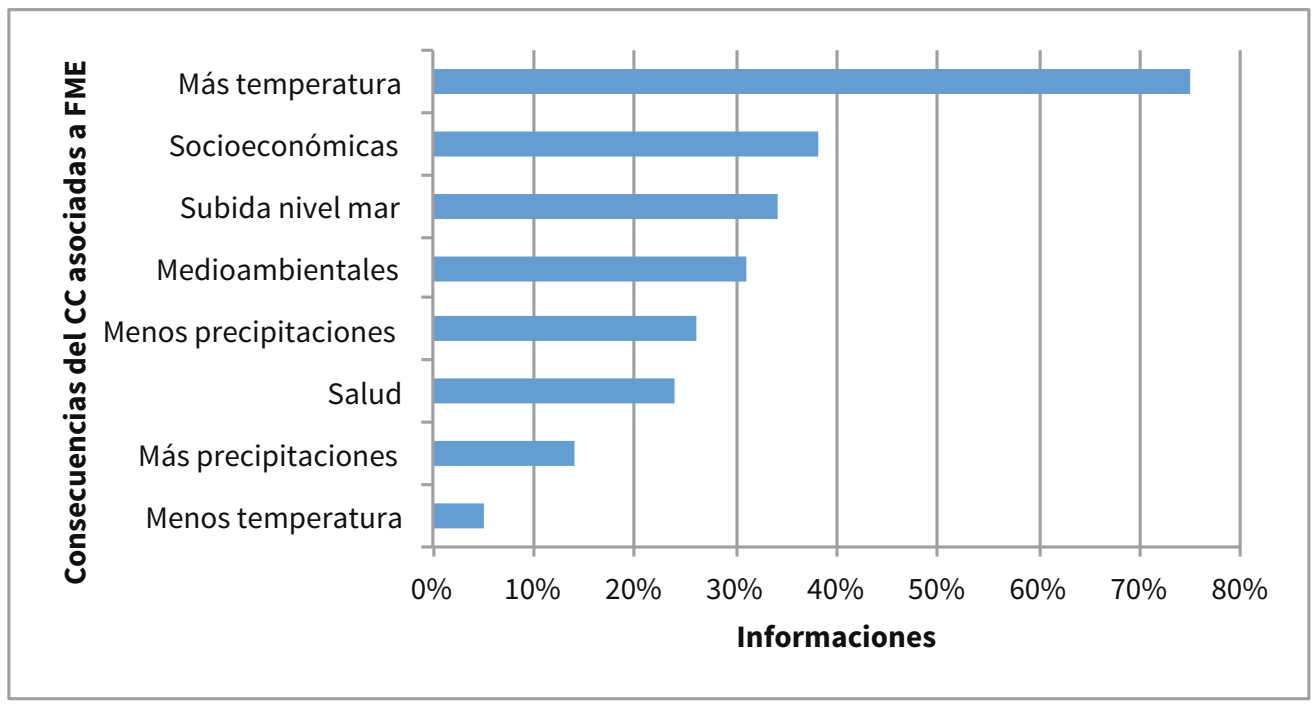

Fuente: Elaboración propia a partir de la base de datos utilizada en el análisis de Lopera (2013).

\section{Evolución de los FME en la cobertura informativa del CC por años y por periódicos}

Desde una perspectiva diacrónica, la figura 6 muestra la evolución de la presencia de los FME en las informaciones sobre la ciencia del clima en la prensa española en el periodo analizado. Como el volumen de informaciones/

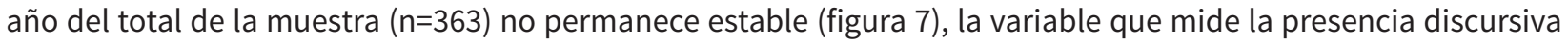
de los FME está representada en cifras porcentuales anuales para que su valor no se vea afectado por el volumen total de atención mediática de cada ejercicio, sino que sea proporcional. Volviendo a la figura 6 se comprueba que la recurrencia a los FME no es regular a lo largo del tiempo, sino que experimenta importantes oscilaciones. Atendiendo a dichas oscilaciones, se establecen tres fases diferenciadas que parecen marcar un ciclo de atención mediática (Downs, 1972): a) fase de descenso (2000-2003); b) fase de aumento (2004-2007); c) nueva fase de descenso (2008-2010). 
Figura 6. Distribución de la submuestra analizada (Informaciones FME) por años ( $\mathrm{n}=124)$

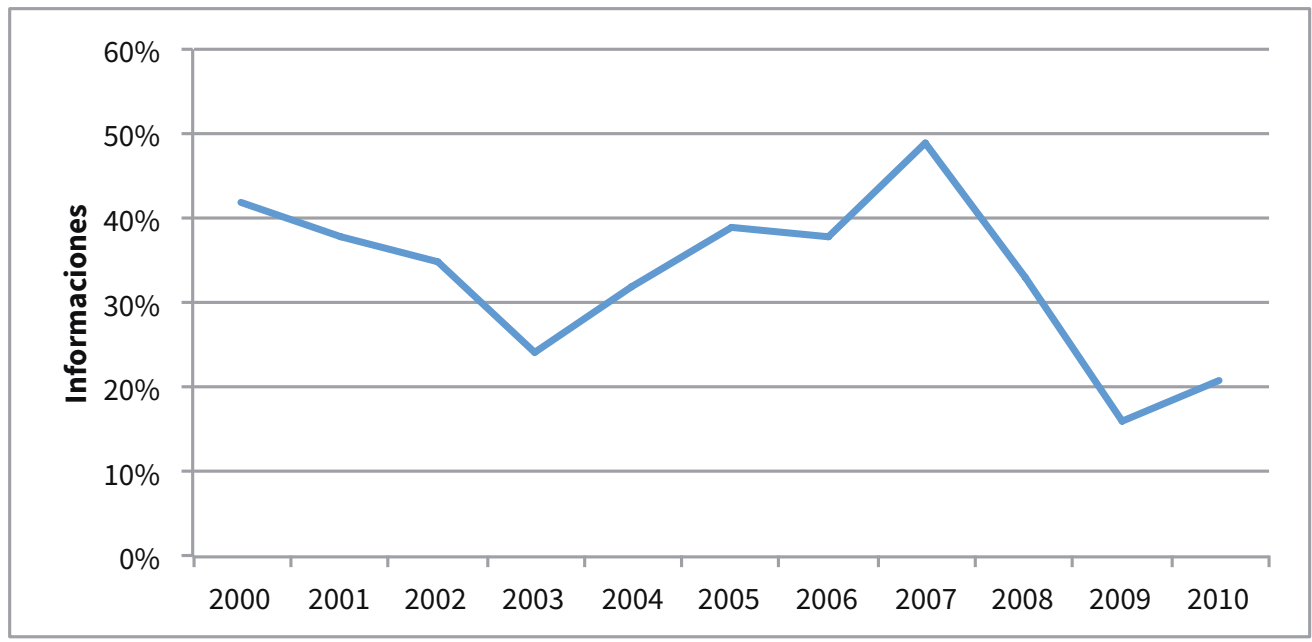

Fuente: Elaboración propia a partir de la base de datos utilizada en el análisis de Lopera (2013).

Figura 7. Distribución del total de la muestra de informaciones sobre la ciencia del clima por años (n=363)

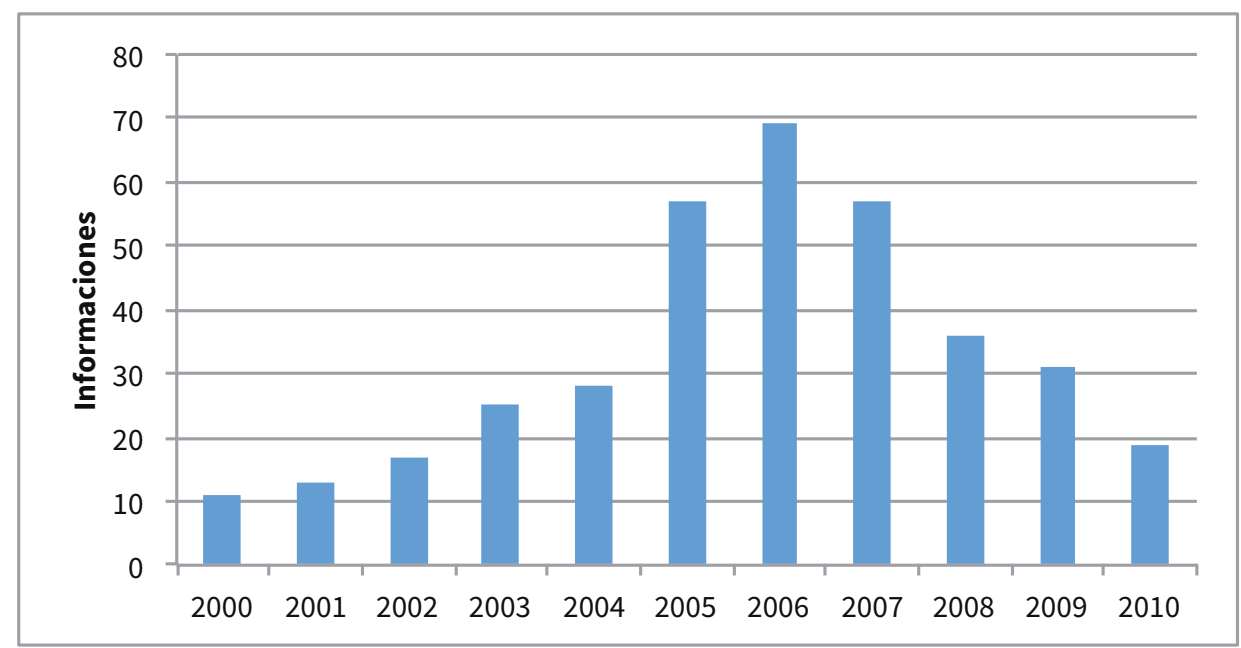

Fuente: Lopera (2013).

Atendiendo a las tres fases marcadas en rojo en la figura 6, tenemos que a principios de la década pasada alrededor del $40 \%$ de las informaciones sobre la ciencia del clima en la prensa escrita española incluía referencias a la ocurrencia de FME como consecuencia del CC, aunque solo cuatro años más tarde dicha cifra se redujo en un 
$20 \%$. Sin embargo, en los siguientes cuatro años (2004-2007) la conexión volvió a ser tomada en consideración, incrementándose su presencia hasta casi el $50 \%$ de los casos en 2007, la cifra más alta de toda la década. Finalmente, de 2008 a 2010, se produjo un nuevo descenso en la presencia de este elemento discursivo que terminó la década presente en solo el $20 \%$ de los casos.

Pero incluso en plena fase de descenso de la recurrencia de los FME como elemento discursivo, a principios de la década aparecieron informaciones que en el titular daban por hecho el nexo entre CC y FME. Este es el caso de El País que tituló "Las lluvias torrenciales desbordan las previsiones del cambio climático" (15/08/2002) para informar de las inundaciones que se producían en Alemania, Austria y Polonia. El desarrollo de la noticia denota mayor rigor periodístico y análisis de los hechos de los que se informa cuando se explica que estos desastres suponen un "refuerzo para los ecologistas aunque no exista una certeza absoluta de que las riadas que están devastando amplias zonas de Europa Central se originen por el cambio climático".

Por periódicos, las diferencias en la frecuencia con que cada medio conecta los FME con el CC son mucho menos notorias que en el análisis diacrónico (figura 8). Si por años se han identificado diferencias de hasta el $30 \%$, el mayor contraste entre periódicos es del $13 \%$ y se encuentra entre los valores de $A B C$ ( $26 \%$ ) y los del diario económico Expansión (39\%). No obstante, hay que destacar que los rotativos que menor atención mediática dedican a la ciencia del clima - Levante y Expansión - (Lopera, 2013), son precisamente los que con mayor frecuencia incluyen la conexión entre CC y FME.

Figura 8. Distribución de la submuestra de informaciones sobre FME por periódicos ( $n=124)$

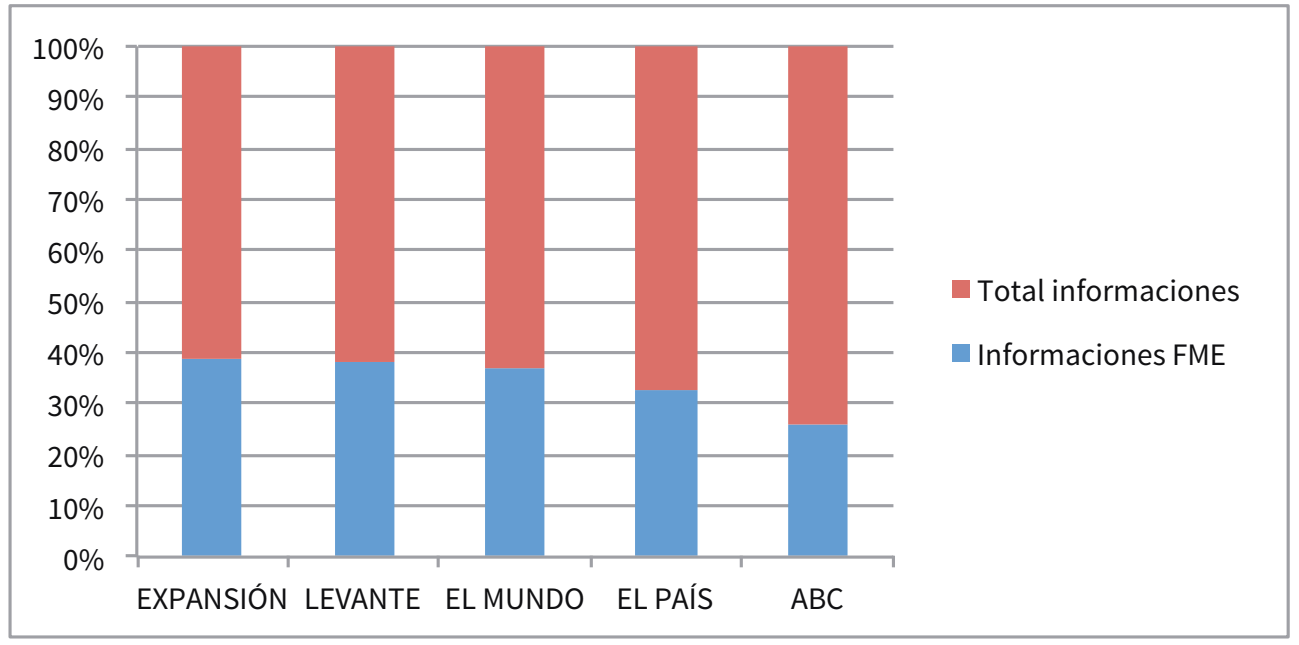

Fuente: Elaboración propia a partir de la base de datos utilizada en el análisis de Lopera (2013). 


\section{Recurrencia de los FME en función de otras variables: fuentes, escalas espacial y temporal, y enmarcados de la ciencia del clima}

No se han encontrado diferencias importantes en el uso de fuentes de información entre el conjunto de la muestra y en las noticias en que se alude a los FME, aunque en este último caso los periodistas citaron con mayor frecuencia a las fuentes próximas al IPCC, la ONU o las ONG (figura 9).

\section{Figura 9. Tipos de fuentes de información}

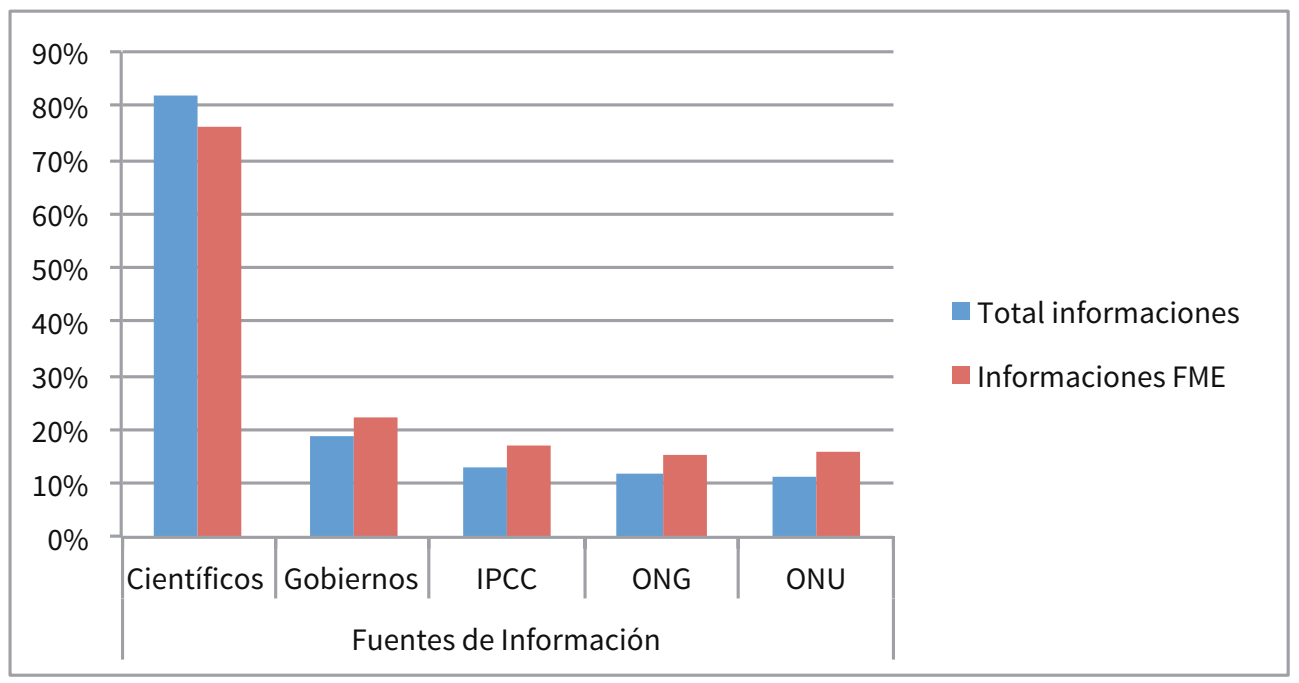

Fuente: Elaboración propia a partir de la base de datos utilizada en el análisis de Lopera (2013).

Pero en las noticias también se encuentran referencias a organismos oficiales de carácter nacional, como la Agencia Estatal de Meteorología, a la que los periódicos acuden para contrastar si determinadas anomalías en las temperaturas y en las precipitaciones pueden considerarse "condiciones meteorológicas excepcionales" y si dichas circunstancias se deben o no al CC. Así sucedió en agosto de 2002, cuando ABC informó:

[...] El mes de agosto de 2002 está siendo de todo menos típico: lluvias torrenciales, bajadas bruscas y repentinas de las temperaturas... En definitiva, un tiempo propio de los meses de septiembre u octubre que está amargando el verano a la mayoría de las personas (ABC, 11/08/2002).

En este caso, las fuentes de información especializadas contestaron al periódico que "hablar de cambio climático es un poco prematuro [...], aunque no se tiene constancia de que se hubieran dado tantos fenómenos meteorológicos extraños a la vez", como nevadas, granizo y trombas de agua en pleno verano.

En cuanto a las escalas espacial y temporal de las consecuencias del CC en las informaciones en las que se hace referencia a los FME, estas siguen la tónica general del conjunto de la muestra aunque se detecta una mayor tendencia a identificar geográfica y temporalmente dichos fenómenos (figuras 10 y 11). La hipótesis de la hipermetropía ambiental (Uzzel, 2000), que podría definirse como la propensión de los seres humanos a alejar en el tiempo y el espacio las consecuencias de los problemas medioambientales como una estrategia para reducir su respon- 
sabilidad en ellos, solo se cumple en el caso de la escala espacial (figura 10), mientras que en el caso de la escala temporal existe un equilibrio entre las informaciones que se ocupan de los FME que ocurren en la actualidad y las que los mencionan como una amenaza para el futuro (figura 11).

Figura 10. Caracterización espacial de las consecuencias en las informaciones sobre FME

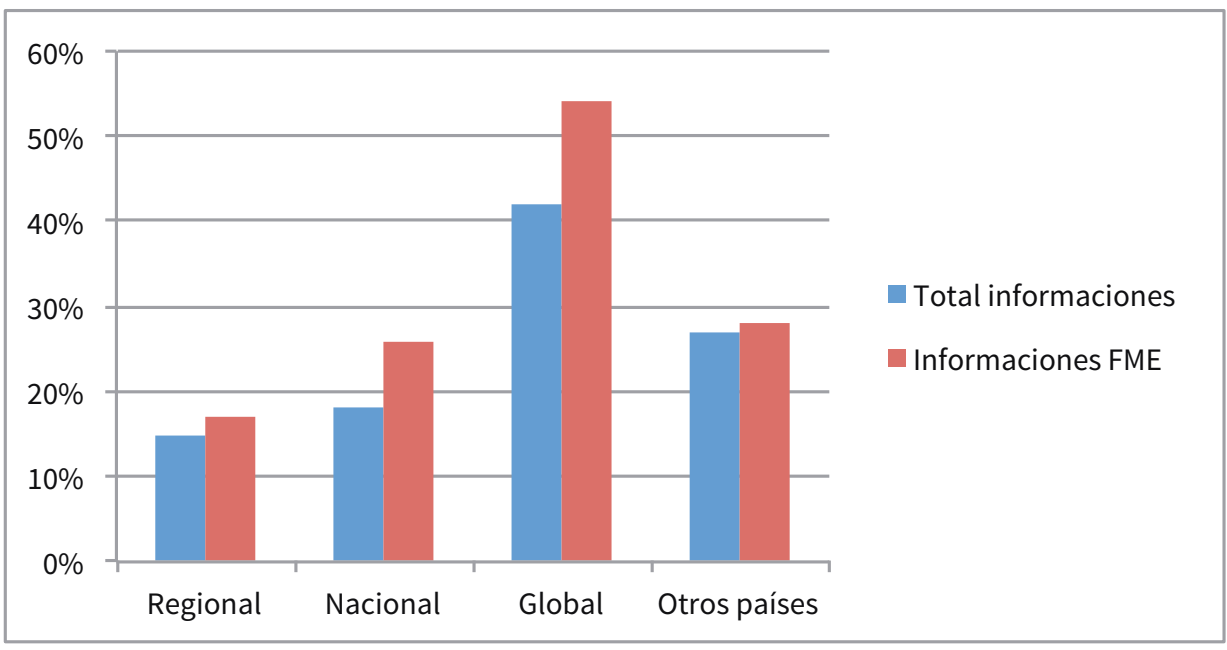

Fuente: Elaboración propia a partir de la base de datos utilizada en el análisis de Lopera (2013).

Figura 11. Caracterización temporal de las consecuencias en las informaciones sobre FME

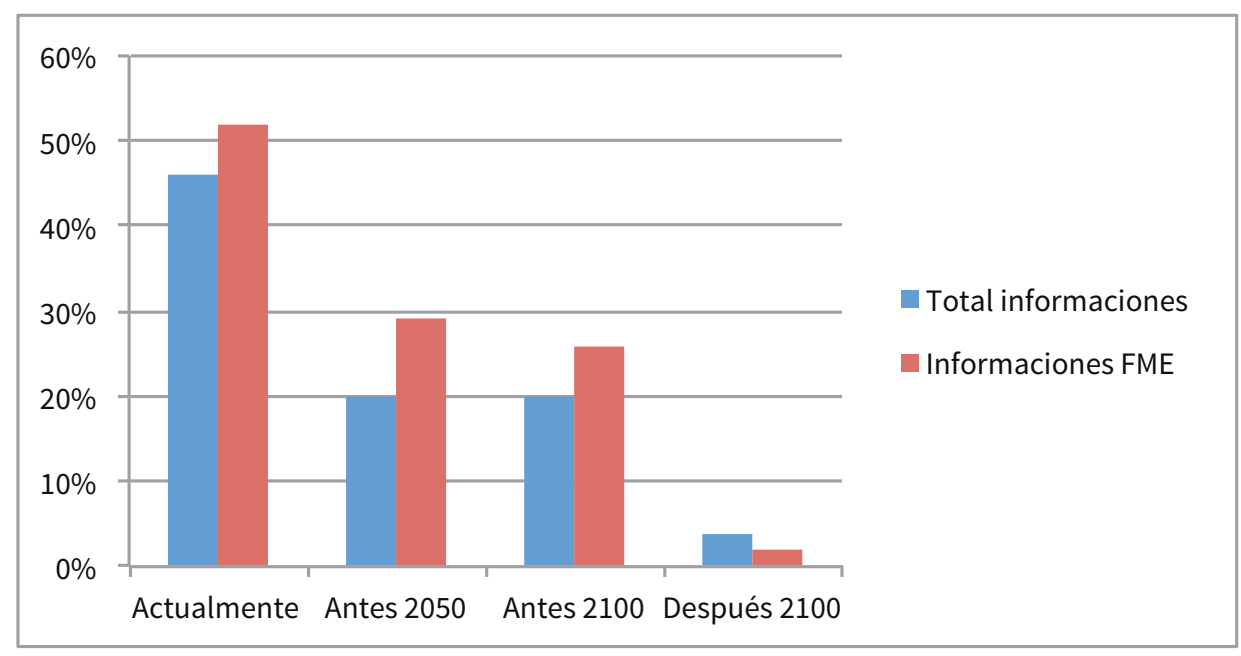

Fuente: Elaboración propia a partir de la base de datos utilizada en el análisis de Lopera (2013). 
En lo que respecta a los enmarcados específicos de la ciencia del clima como ciencia sólida o ciencia incierta, ha sido una sorpresa constatar que apenas existen diferencias entre el enmarcado del conjunto de la muestra y el de las informaciones con alusiones a FME: tanto en la muestra como en la submuestra solo alrededor del $10 \%$ de los casos presenta la ciencia del clima como una ciencia controvertida e incierta, siendo la cifra ligeramente superior en el caso de la muestra general (figura 12).

Figura 12. Enmarcados de la ciencia del clima en las informaciones sobre FME

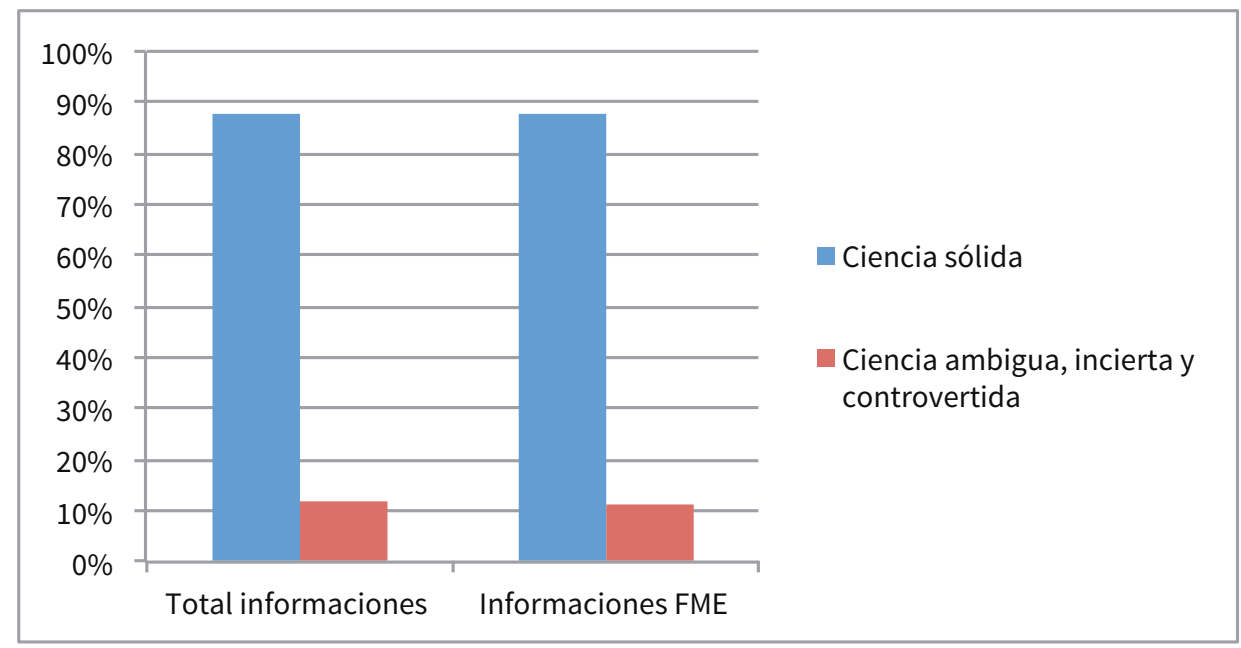

Fuente: Elaboración propia a partir de la base de datos utilizada en el análisis de Lopera (2013).

Una de las fórmulas para crear incertidumbre científica es la simetría de fuentes que consiste en citar a dos o más fuentes de información con versiones enfrentadas a las que se otorga el mismo peso (Boykoff y Boykoff, 2004). Encontramos un ejemplo de ello en Expansión que, a mediados de la década pasada, puso el foco sobre el aumento de los huracanes en el mar Caribe y en Florida y su posible relación con el CC (Expansión, 31/01/2005). En esta noticia se utiliza la simetría de fuentes de información de tal manera que, mientras que para la Organización Meteorológica Mundial "existe un vínculo muy claro entre el cambio climático y el aumento del número de desastres naturales", los científicos del Gobierno estadounidense se muestran divididos al respecto. Dicha información finaliza con la siguiente conclusión: "Este es el problema del cambio climático: por el momento, no podemos afirmar con seguridad que se esté produciendo".

\section{Discusión}

Estudios previos han revelado que las consecuencias del CC son uno de los aspectos más tratados por los medios de comunicación españoles, tanto en la prensa (Lopera, 2013) como en la televisión (Teso-Alonso, 2016), en comparación con otros países (McComas y Shanahan, 1999; Trumbo, 1996). La menor atención dedicada a las causas y 
a las soluciones ha sido considerada una de las carencias importantes de la comunicación social del riesgo climático en España (Lopera y Moreno, 2014b), junto con su escaso tratamiento informativo en comparación con otros temas de la agenda (Teso-Alonso, 2016). Desde la aproximación de la teoría de la amplificación social del riesgo (Renn et al., 1992), este artículo plantea que esa tendencia de los medios a centrarse en las consecuencias podría suponer una ventana de oportunidad para popularizar el posible nexo causal entre CC y FME y, por ende, aumentar la sensación de vulnerabilidad y acelerar la acción. Al explorar esta idea, este trabajo constata que en las últimas décadas se ha producido un aumento en la frecuencia y/o intensidad de los FME y que más de un tercio de las informaciones sobre la ciencia del clima en la prensa española incorporan discursivamente el citado nexo CC-FME.

Según los resultados obtenidos, el aumento de las temperaturas y la ocurrencia de FME es la combinación más recurrente con que los periódicos españoles ilustran el impacto del CC en la primera década del siglo XXI. Este resultado parece coherente con los datos de la Organización Meteorológica Mundial sobre los FME en la década 2001-2010 en todo el mundo (OMM, 2013) pues, según este informe, el $44 \%$ de los países reportó récord de temperatura máxima diaria en tal periodo y, precisamente debido a las olas de calor, también aumentó la pérdida de vidas humanas en un $2300 \%$ en comparación con la década anterior (OMM, 2013, pp. 7-8). Estas cifras globales recogen la fuerte ola de calor que sufrió Europa (y España) en el verano de 2003, con un coste de 66000 vidas. En el mismo periodo, pero en el contexto español, los FME más frecuentes fueron las inundaciones, aunque el mayor número de víctimas mortales se debió a las olas de calor y las mayores pérdidas económicas, a los incendios forestales y las tormentas (EM-DAT, 2016).

Cabe destacar que esta sensación de exposición y vulnerabilidad ante la ocurrencia de FME parece haber permeado en la sociedad española a tenor de los resultados demoscópicos (Meira et al., 2013). Según esta encuesta, una gran mayoría de la ciudadanía considera muy probable o bastante probable la sucesión de periodos de sequía más frecuentes ( $86 \%$ ), el aumento de los incendios forestales ( $85 \%$ ), el aumento significativo de las temperaturas (84 \%) y el incremento de las inundaciones (75 \%) (Meira et al., 2013, pp. 60 y ss.).

El hecho de que de 2004 a 2007 se obtenga una mayor atención mediática a los FME en la cobertura informativa de la ciencia del clima (figura 6) contribuye a situar en un contexto de CC una serie de eventos adversos que tuvo lugar en esos años, como la fuerte ola de calor de 2003 en Europa y España ya citada, el aumento del riesgo de inundaciones en Centroeuropa y Gran Bretaña, dos duras temperadas de huracanes en el Atlántico en 2004 y 2005 y la intensificación del fenómeno de El Niño y La Niña en el Pacífico (Van Aalst, 2006). Por otra parte, resulta preocupante que justo los dos periódicos que menor atención mediática han dedicado a la ciencia del clima en el periodo analizado -Expansión y Levante - (Lopera, 2013) sean precisamente los que recurren con mayor frecuencia a la conexión CC-FME, como si estos eventos extremos fueran un "gancho informativo", tal y como recoge la literatura al respecto (Boykoff y Roberts, 2007).

En definitiva, y a la luz de este conjunto de datos, cabría considerar la cobertura informativa del CC como vector de popularización del posible nexo causal entre CC y FME y, a reglón seguido, plantearse si el abordaje del CC, en cuanto al diseño de políticas públicas ambiciosas y aceptación social de dichas medidas, se puede beneficiar por el hecho de que los medios incluyan la conexión CC-FME. Según Gavin et al. (2011), este sería un camino no exento de riesgos para la praxis política y para la credibilidad de la ciencia, ya que en los años o periodos en que descendiera, por ejemplo, el número de inundaciones, los sectores críticos o escépticos capitalizarían dicha ambigüedad. 


\section{Conclusiones}

La ocurrencia de FME a nivel global ha aumentado en las últimas décadas según los registros disponibles. La posible relación causal entre CC y FME es un elemento discursivo bien imbricado en la cobertura informativa de la ciencia del clima en la prensa española presente en tres de cada diez noticias del periodo 2000-2010. Ahora bien, las oscilaciones en la incorporación de este elemento al discurso público del CC no depende tanto del medio informativo al que nos expongamos como del año en que lo hagamos. Tal es así que las alusiones a FME son más frecuentes, en cifras absolutas y relativas, a partir de la alarma que produjo la ola de calor que sufrió España y Europa en el verano de 2003 y de la devastación que acarreó el huracán Katrina en la costa Este de Estados Unidos en 2005.

El aumento de facto de la frecuencia y/o intensidad de los FME en todo el mundo, con su correspondiente relato mediático de "quiebra del acontecer", y la incorporación discursiva del nexo CC-FME en la información periodística pueden estar creando un caldo de cultivo en el que a los ciudadanos cada vez les resulta menos contraintuitivo relacionar la ocurrencia de FME como los efectos del CC.

Aunque este trabajo solo pretende ser una primera aproximación que habrá que contrastar con otras muestras y en otros marcos temporales, se ha comprobado que la conexión causal CC-FME está presente en la agenda mediática y, por tanto, ha formado parte de la experiencia social de dicho riesgo, tal y como lo recogen las encuestas de años pasados (Meira et al., 2013).

En futuras investigaciones a desarrollar en los próximos años habría que monitorizar si la existencia de ese caldo de cultivo puede funcionar como facilitador y acelerador de la toma de decisiones políticas para hacer frente al CC con objeto de reducir la exposición y vulnerabilidad ante estos eventos adversos. Para ello, habrá que seguir muy de cerca el desarrollo y aplicación del Acuerdo de París, firmado a finales de 2015, en la política nacional e internacional.

\section{Referencias}

1. Almarza Mata, C. (2001). Fenómenos climatológicos extremos. Física y Sociedad, (12), 38-41.

2. Biernacki, W., Bokwa, A., Domanski, B., Dzialek, J., Janas, K., y Padlo, T. (2008) Mass Media as a Source of Information About Extreme Natural Phenomena in Southern Poland. En A. Carvalho (Ed.), Communicating Climate Change: Discourses, Mediations and Perceptions (pp. 190-200). Braga: Centro de Estudios de Comunicaçao e Sociedade, Universidad do Minho

3. Boykoff, M. T., y Boykoff, J. M. (2004). Balance as Bias: Global Warming and the US Prestige Press. Global Environment Change, (14), 125-136.

4. Boykoff, M. T., y Roberts, J. T. (2007). Media Coverage of Climate Change: Current Trends, Strengths, Weaknesses: Human Development Report 2007/2008, Fighting Climate Change: Human Solidarity in Divided World, Occasional Paper, 2007/3.

5. Brulle, R. J., Carmichael, J., y Jenkins, J. C. (2012). Shifting Public Opinion on Climate Change: An Empirical Assessment of Factors Influencing Concern over Climate Change in the U.S., 2002-2010. Climate Change, 114 (2), 169-188. 


\section{DISERTACIONES}

ESTUDIOS

Anuario electrónico
ISSN: $1856-9536$

Doi: http://dx.doi.org/10.12804/revistas.urosario.edu.co/disertaciones/a.4630

Volumen 10, Número 2 / Julio-diciembre 2017

Versión PDF para imprimir desde

http://revistas.urosario.edu.co/index.php/disertaciones

6. Carle, J. (2015). Climate Change Seen as Top Global Threat. Pew Research Center. Recuperado de http:// www.pewglobal.org/files/2015/07/Pew-Research-Center-Global-Threats-Report-FINAL-July-14-2015.pdf

7. Congleton, R. D. (2006). The Story of Katrina: New Orleans and the Political Economy of Catastrophe. Public Choice, 127, 5-30.

8. CRED. (2016). The Human Cost of Weather Related Disasters 1995-2015. Recuperado de http://www.cred. be/HCWRD

9. Crespo, E., Revilla, J. C., y Elizaga, F. (2014). Meteorología y sociedad: uso y evaluación de la información meteorológica. Comunicación y Sociedad, 27 (2), 169-194.

10. Changnon, S. A. (2003). Shifting Economic Impacts from Weather Wxtremes in the United States: A Result of Societal Changes, Not Global Warming. Natural Hazards, 29 (2), 273-290.

11. De Rueda Úbeda, A. (2015). El discurso político y el tratamiento periódistico del cambio climático en la prensa española durante las conferencias de las partes de Naciones Unidas: de Copenhague (2009) a Varsovia (2013). Tesis doctoral. Departamento de Comunicación e Información Periodística. Valencia: Universidad Cardenal Herrera CEU de Valencia.

12. Downs, A. (1972). Up and Down with Ecology: The "Issue-Attention Cycle". Public Interest, 28, 38-50.

13. EM-DAT. (2016). Base de Datos Internacional sobre Desastres. Recuperado de http://www.emdat.be/

14. Fernández Reyes, R., y Mancinas Chávez, R. (2013). Medios de comunicación y cambio climático. Sevilla: Fénix Editora.

15. Fernández Reyes, R., Piñuel-Raigada, J. L., y Vicente Mariño, M. (2015). La cobertura periodística del cambio climático y del calentamiento global en El País, El Mundo y La Vanguardia. Revista Latina de Comunicación Social, (69), 122-140.

16. Ford, J. D., y King, D. (2015). Coverage and Framing of Climate Change Adaptation in the Media: A Review of Influential North American Newspapers During 1993-2013. Environmental Science and Policy, 48, 137-146.

17. Gavin, N. T., Leonard-Milsom, L., y Montgomery, J. (2011). Climate Change, Flooding and the Media in Britain. Public Understanding of Science, 20 (3), 422-438.

18. González Alcaraz, L. (2014). El framing como legitimación de la política climática. Encuadres del cambio climático en la prensa argentina y brasileña durante las conferencias de Doha y Varsovia. Disertaciones, 7 (1), 224-259.

19. IPCC. (2014). Climate Change 2014. Impacts, Adaptation and Vulnerability. Summary for Policymakers. Recuperado de https://ipcc-wg2.gov/AR5/images/uploads/IPCC_WG2AR5_SPM_Approved.pdf

20. Krippendorff, K. (1990). Metodología del análisis de contenido: teoría y práctica. Barcelona: Ediciones Paidós.

21. León, B. (2014). Periodismo, medios de comunicación y cambio climático. Salamanca: Comunicación Social Ediciones y Publicaciones.

22. León Gross, T., y Ruiz Sinoga, J. D. (2012). Impacto mediático de los fenómenos medioambientales: la sequía en el sur de España durante el último medio siglo y su cobertura en prensa. Estudios sobre el Mensaje Periodístico, 18 (2), 769-790.

23. Linnenluecke, M. K., y Griffiths, A. (2010). Beyond Adaptation: Resilience for Business in Light of Climate Change and Weather Extremes. Business \& Society, 49 (3), 477-511. 
24. Linnenluecke, M. K., Stathakis, A., y Griffiths, A. (2011). Firm Relocation as Adaptative Response to Climate Change and Weather Extremes. Global Environmental Change, (21), 123-133.

25. Lopera, E. (2013). La comunicación social de la ciencia del clima en la prensa española: texto y contexto. Tesis doctoral. Departamento de Teoría de los Lenguajes y Ciencias de la Comunicación. Universidad de Valencia. Madrid: Colección Documentos Editorial CIEMAT.

26. Lopera, E., y Moreno, C. (2014a). The Iconographic Discourse of Climate Science, Examined through the Press Coverage of Spanish Daily Newspapers. Paper presented at the 13th International Public Communication of Science and Technology Conference, Salvador de Bahía, Brasil.

27. Lopera, E., y Moreno, C. (2014b). The Uncertainties of Climate Change in Spanish Daily Newspapers: Content Analysis of Press Coverage from 2000 to 2010. JCOM, 01, A02.

28. Lozano Ascencio, C. (2013). El cambio climático en los telediarios: alusiones a la catástrofe en tiempos de calma. Disertaciones, 6 (1), 124-140.

29. Lozano Ascencio, C., Piñuel Raigada, J. L., y Gaitán Moya, J. A. (2012). Construcción social y mediática de la incertidumbre: discursos en torno a las quiembras del acontecer. Prisma Social, 8, 380-413.

30. McComas, K., y Shanahan, J. (1999). Telling Stories About Global Climate Change - Measuring the Impact of Narratives on Issue Cycles. Communication Research, 26 (1), 30-57.

31. Meira, P. A., Arto, M., Heras, F., Iglesias, L., Lorenzo, J. J., y Montero, P. (2013). La respuesta de la sociedad española ante el cambio climático 2013. Madrid: Fundación Mapfre.

32. Meira, P. A., Arto, M., Heras, F., y Montero, P. (2011). La sociedad ante el cambio climático. Conocimientos, valoraciones y comportamientos en la población española 2011: Fundación Mapfre.

33. Meira, P. A., Arto, M., y Montero, P. (2009). La sociedad ante el cambio climático. Conocimientos, valoraciones y comportamientos en la población española: Fundación Mapfre.

34. Nerlich, B., y Jaspal, R. (2014). Images of Extreme Weather: Symbolising Human Responses to Climate Change. Science as Culture, 23 (2), 253-276.

35. OMM. (2013). The Global Climate 2001-2010. A Decade of Climate Extremes. Recuperado de http://library. wmo.int/pmb_ged/wmo_1119_en.pdf

36. ONISDR. (2015). Global Assessment Report on Disaster Risk Reduction 2015. Recuperado de http://www. preventionweb.net/english/hyogo/gar/2015/en/gar-pdf/GAR2015_EN.pdf

37. Padrón Castañeda, N., y Barranco Retes, J. (2014) Cambio climático e incendios de 5a generación. En J. C. Santamarta Cerezal, L. E. Hernández Gutiérrez y M. P. Arraiza Bermúdez-Cañete (eds.), Riesgos naturales y cambio climático (pp. 81-89). Madrid: Colegio de Ingenieros de Montes.

38. Pasquare, F. A., y Oppizzi, P. (2012). How Do the Media Affect Public Perception of Climate Change and Geohazards? An Italian Study. Global and Planetary Change, 90-91, 152-157.

39. Piñuel Raigada, J. L., Águila Coghlan, J. C., Teso-Alonso, G., Teso Alonso, G., Vicente Mariño, M., y Gaitán Moya, J. A. (2012). Comunicación, controversias e incertidumbres frente al consenso científico acerca del cambio climático (Sociedad Latina de Comunicación Social - edición no venal - La Laguna (Tenerife), 2012 - Creative Commons ed. (30) Cuadernos Artesanos de Latina.

40. Renn, O. (1991). Risk Communication and the Social Amplification of Risk. En R. E. Kasperson y P. J. M. Stallen (eds.), Communicating Risk to the Public: International Perspectives (pp. 234-234). Londres: Klower. 
41. Renn, O., Burns, W. J., Kasperson, J. X., Kasperson, R. E., y Slovic, P. (1992). The Social Amplification of Risk: Theoretical Foundations and Empirical Applications. Journal of Social Issues, 48 (4), 137-160.

42. Riffe, D., Lacy, S., y Fico, F. (2005). Analyzing Media Messages Using Quantitative Content Analysis in Research. Mahwah, N. J.: Lawrence Erlbaum Associates.

43. Sánchez Calero, M. L. (2005). La información meteorológica como servicio. Madrid: Ministerio de Medio Ambiente.

44. Schaefer, M. S., Ivanova, A., y Schmidt, A. (2014). What Drives Media Attention for Climate Change? Explaining Issue Attention in Australian, German and Indian Print Media from 1996 to 2010. International Communication Gazette, 76 (2), 152-176.

45. Scheffer, M., Carpenter, S., Foley, J. A., Folke, C., y Walker, B. (2001). Catastrophic Shifts in Ecosystems. Nature, (418), 591-596.

46. Teso-Alonso, G. (2016). Comunicación y representación del cambio climático: el discurso televisivo y el imaginario de los jóvenes españoles. Tesis doctoral, Universidad Complutense de Madrid.

47. Trumbo, C. (1996). Constructing Climate Change: Claims and Frames in US News Coverage of an Environmental Issue. Public Understanding of Science, 5, 269-283.

48. Ungar, S. (1999). Is Strange Weather in the Air? A Study of US National Network News Coverage of Extreme Weather Events. Chlimate Change, 41, 133-150.

49. Uzzel, D. L. (2000). The Psycho-Spatial Dimension of Global Environmental Problems. Journal of Environmental Psychology, 20, 307-318.

50. Van Aalst, M. K. (2006). The Impacts of Climate Change on the Risk of Natural Disasters. Disaster, 1 (30), 5-18.

51. Wilson, K. M. (2002). Forecasting the Future. How Television Weathercasters' Attitudes and Beliefs About Climate Change Affect their Cognitive Knowledge on the Science. Science Communication, 24 (2), 246-268.

52. World Economic Forum. (2016). Global Risks 2016 - Eleven Edition. Ginebra: World Economic Forum.

53. WWA. (2016). World Weather Attribution Project. Recuperado de http://www.climatecentral.org/wwa

54. Yan, Y., y Bissell, K. (2015). The Sky is Falling. Predictors of News Coverage of Natural Disasters Worldwide. Comunication Research, 0093650215573861. 